\title{
A framework for modeling the cathode fall illustrated with a single beam model
}

\author{
T. J. Sommerer and I. E. Lawier \\ Department of Physics, University of Wisconsin, Madison, Wisconsin 53706 \\ W. N. G. Hitchon \\ Deparment of Electrical and Computer Engineering, University of Wisconsin, Madison, \\ Wiscortsin 53706
}

(Received 21 March 1988; accepted for publication 3 May 1988)

\begin{abstract}
A framework for a model of the cathode fall region of a dc glow discharge is presented, and a simple model is solved as an illustration. An extremum condition independent of the model is placed on the electric field behavior to produce a unique solution that agrees with experiment. The zeroth and second moments of the Boltzmann equation are solved for the electrons with a self-consistent electric field. A single-beam model with only two parameters (number density and beam velocity) is assumed for the electron distribution function. Ion motion is modeled with a parametric fit to known ion mobilities. The model is solved for conditions corresponding to the experimental results and to Monte Carlo simulations of Doughty, Den Hartog, and Lawler [Phys. Rev. Lett. 58, 2668 (1987)]. The results are in good qualitative and "factor-of-two" quantitative agreement with the published results.
\end{abstract}

\section{INTPODUCTION}

The cathode fall region of glow discharges has come under increased scrutiny with the development of laser diagnostics for the region and with the application of glow discharges in plasma processing. The cathode fall is the source of instabilities in many gas-phase pulsed-power devices. The region is of fundamental interest because the electrons in it are not in hydrodynamic equilibrium due to the proximity of the cathode boundary, which absorbs ions and emits electrons, and due to the strong electric field gradients. The introduction of a recent paper by Bayle, Vacquie, and Bayle includes an excellent list of references reviewing the latest experimental and theoretical efforts to understand the cathode fall.

This paper presents an approach to modeling the cathode fall, including a necessary extremum condition that specifies the correct electric field behavior. A single beam model is then used to illustrate the approach, a model that is simple and yet able to produce results which reflect many of the results from both Monte Carlo simulations (which are very time consuming) and various experiments.

\section{ROOEL}

\section{A. Physical assumptions}

Various simplifying physical assumptions have been made to make the problem tractable. Most of these are consistent with the experiments and simulations of Doughty and co-workers ${ }^{2,3}$ and Den Hartog, Doughty, and Lawler, ${ }^{4}$ with whose work comparisons will be made.

The entire solution is independent of time.

The discharge exists between two parallel plates and extends radially to infinity. The electron distribution therefore only depends on one spatial coordinate (the distance $z$ from the cathode). The positive $z$ direction is taken to be in the direction from the cathode to the anode.
Electron losses due to radial difusion are negligible.

The electric field depends only on the $z$ coordinate and is directed along the $z$ axis. Effects of magnetic fields are ignored.

The gas is assumed to be weakly ionized. Only electronneutral collisions and singly-ionized ions are important.

The neutral background gas is assumed to be "cold" relative to the electron temperature. If distribution function is therefore 2 delta function at zero energy: $N \delta(v)$.

The elastic collision cross section is replaced with the momentum transfer cross section:

$$
\sigma_{\mathrm{MT}}(\tau)=\int_{4 \pi}(1-\cos \theta)\left(\frac{d \sigma}{d \Omega}\right)_{\mathrm{EL}} d \Omega
$$

where $\theta$ is the scattering angle and $\tau$ is the electron kinetic energy.

\section{B. The mathematlas mocel}

A model of the electron transport and kinetics should be based on the Boltzmann equation:

$$
\frac{\partial f}{\partial t}+\mathrm{v} \cdot \nabla_{\mathrm{r}} f+a \cdot \nabla_{\mathrm{V}} f=C
$$

where $f=f(\mathbf{r}, \mathbf{v}, t)$ is the electron distribution function, $a$ is the acceleration vector due to the local fields, and $C$ represents the collision operator. The gradients are to be taker with respect to real and velocity space in the second and third terms, respectively.

From the physical assumptions listed above, the full Boltzmann equation presented can be simplified greatly. Time independence removes the first term. Plane parallel spatial symmetry reduces the spatial gradient term to one derivative with respect to $z$. The velocity gradient, usually taken in a spherical velocity space, has no dependence on the azimuthal angle $\phi$. The acceleration can be explicitly written as $=-(e E / m) \hat{\mathbf{z}}$, where $e$ and $m$ are the electronic 
charge and mass, respectively, and $E$ is the electric field along the $z$ axis. The electric field $E$ is directed toward the cathode in the cathode fall; therefore, $E<0$. The electronic charge $e$ will be taken to be positive, and the negative sign explicitly displayed.

In order to use the moment equation approach, the form of the distribution must be assumed. In keeping with the desire to produce a model that can be quickly solved numerically, the assumed distribution function is that of a monoenergetic beam of electrons moving along the axis of the discharge:

$$
f(z, v)=n(z) \delta\left[v_{z}-u(z)\right] \delta\left(v_{x}\right) \delta\left(v_{y}\right) .
$$

Here, $n$ is the number density of electrons and $u$ is the beam velocity. There would appear to be no simpler suitable distribution function. This distribution has only two free parameters, and therefore requires only two equations for solution. In contrast to the usual requirement that electron number, momentum, and energy all balance, ${ }^{5}$ we can only require two of these to balance. Phelps, Jelenkovic, and Pitchford found that balancing number density and beam energy (rather than momentum) gave superior results"; that was found to be the case for this study, also. This is perhaps not surprising; elastic scattering will be shown to be much more important in the momentum balance equation, while it is suppressed by the factor $m / M$ in the energy balance equation. The mass of a neutral gas atom is $M$. Regions such as the cathode fall have very high electric fields and mean electron energies; hence, forward elastic scattering dominates.

Phelps and co-workers ${ }^{6}$ have investigated a similar single beam model and calculated the moment equations. If the assumed distribution function ( 3 ) is now substituted into the Boltzmann Eq. (2) and the result integrated over all velocities, the electron number balance equation is generated:

$$
\frac{d(u n)}{d(N z)}=n u \sigma_{\mathrm{ion}}(u)
$$

If the Boltzmann Eq. (2) is multiplied by $v^{2}$ before the distribution (3) is inserted and integrated, the energy balance equation is obtained:

$$
\begin{aligned}
\frac{d\left(n u^{3}\right)}{d(N z)}= & -\frac{2 n u e E}{m N}-u n\left(u_{\mathrm{ion}}^{2} \sigma_{\mathrm{ion}}(u)\right. \\
& \left.+\sum_{k} u_{k}^{2} \sigma_{k}(u)+\frac{2 m}{M} u^{2} \sigma_{\mathrm{M} \mathrm{r}}(u)\right)
\end{aligned}
$$

where $\sigma_{k}(u)$ is the total cross section for the $k$ th excitation process, $\sigma_{\text {ion }}(u)$ is the total ionization cross section, and $\sigma_{\mathrm{MT}}(u)$ is the momentum transfer cross section for elastic collisions, described above. The threshold excitation and ionization electron energies are given by $\tau_{k}=m u_{k}^{2} / 2$ and $\tau_{\text {ion }}=m t_{\text {ion }}^{2} / 2$.

The number density can be eliminated by combining Eqs. (4) and (5) to obtain a single, uncoupled equation describing the velocity (or energy) of the beam:

$$
\begin{aligned}
\frac{d\left(\frac{1}{2} m u^{2}\right)}{d(N 2)}= & -\frac{e E}{N}-\frac{1}{2} m u_{\mathrm{ion}}^{2} \sigma_{\mathrm{ion}}(u) \\
& -\sum_{k} \frac{1}{2} m u_{k}^{2} \sigma_{k}(u)-\frac{2 m}{M} \frac{1}{2} m u^{2} \sigma_{\mathrm{MT}}(u) \\
& -\frac{1}{2} m u^{2} \sigma_{\mathrm{ion}}(u) .
\end{aligned}
$$

The last verm of this equation accounts for an artifact of the beam model--every electron must be moving with the beam velocity. An ionization event removes not only the ionization energy from the beam, but also the energy necessary to accelerate the new electron from rest to the beam energy. If the electric field behavior is known, this equation can be directiy integrated.

One particularly appealing aspect of this beam model is that cross section integration is not necessary. For instance, once the beam velocity is known, it is simple to calculate the effective Townsend ionization coefficient:

$$
\begin{aligned}
\alpha(z) & =\frac{\text { ionization rate }}{\text { electron fux density }} \\
& =\frac{N \int_{0}^{\infty} d v v^{3} \sigma_{\text {ion }}(v) f(z, v)}{\int_{0}^{\infty} d v v^{3} f(z, v)} \\
& =N \sigma_{\text {ion }}(u) .
\end{aligned}
$$

Note that the same simplicity has carried into all other collision terms. Where integrals over cross sections would ordinarily appear, it is necessary only to evaluate the cross sections at a single energy; namely, the beam energy.

Poisson's equation is used to determine the electric field. Once simplified by the assumption that only the $z$ coordinate is important, it becomes

$$
\frac{d E}{d z}=\frac{e}{\epsilon_{0}}\left(n_{+}-n\right) \text {. }
$$

The ion number density, $n_{+}$, will be eliminated by fixing the current density throughout the discharge and using experimentally known ion mobilities.

\section{Treatment of lons}

In order to solve the Boltzmann equation with a selfconsistent electric field, it is necessary to account for the behavior of all charged species. In this work, the ions are assumed to be in hydrodynamic equilibrium with the electric fields present; ion motion can therefore be modeled using the experimentally known mobility of singly charged helium atoms through a neutral helium background. This assumption is supported by Lawler's calculations ${ }^{7}$ of ion equilibration distance in the cathode fall. He found that ions reach $90 \%$ of their equilibrium drift velocity in one to six mean free paths, depending upon the spatial distribution of ionization and the functional form of the electric field. Using the charge exchange cross section of Sinha, Lin, and Bardsley, ${ }^{8}$ the equilibrium distance is found to be only about $5 \%$ of the length of the cathode fall. It is therefore justified to use known mobilities over nearly all of the cathode fall.

A parametric fit was made to the mobility data of $\mathrm{Helm}^{9}$ using the functional form suggested by Frost ${ }^{10}$ : 


$$
\mu_{+}=\frac{\mu_{0}}{\sqrt{1+a|E| / N}} \frac{N_{0}}{N},
$$

where

$$
v_{+}=\mu_{+} E \text {. }
$$

Heim's data covers experimental regimes in the range 10

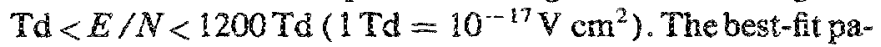
rameters used were $\mu_{0}=11.28 \mathrm{~cm}^{2} \mathrm{~V}^{-1} \mathrm{~s}^{-1}$ and $a=0.01392 \mathrm{Td}^{-1}$ at standard temperature and pressure (273 $\mathrm{K}$ and $1 \mathrm{~atm}$ ), corresponding to a neutral number density of $N_{0}=2.6868 \times 10^{19} \mathrm{~cm}^{-3}$. This same parametrization will be extrapolated to higher values of $E / N$ than Helm tabulated, however. Wannier ${ }^{11}$ showed that for ions in high fields, $\left\langle v_{+}\right\rangle=(2 a / \pi N \sigma)^{1 / 2}$, where $a$ is the acceleration and $\sigma$ is the ion-atom exchange cross section; for ions here, $a=e E / M$. The point is that $v_{+} \propto E^{1 / 2}$ for ions in strong fields, and Frost's parametrization has already included this behavior.

\section{Boundary conditions at the cathode}

The electron number density, beam velocity, and electric field must be known at the cathode to integrate the coupled equations into the cathode fall. The cathode beam velocity is fixed. Monte Carlo simulations tend to assume a fiat distribution of electrons emitted from the cathode, with typical energies less than $10 \mathrm{eV}^{1-4,12,13}$ It would seem convenient to launch all electrons from rest, but this produces an intinite number density of electrons at the cathode, since the current density is assumed constant for all $z$. Here, the beam of electrons was assumed to be ernitted from the cathode with an energy of $2 \mathrm{eV}$-the large cathode fall electric field quickly accelerates the beam to tens or hundreds of $\mathrm{eV}$; the small launch energy is rather arbitrary. The cathode electron number dersity can then be found by combining the ion mobility already described with the definition of the current density, which is fixed throughout the entire discharge:

$$
j=n_{+} e v_{+}-r e u_{2}
$$

and an assumption about the secondary electron emission coefficient $\gamma_{p}$. (The plus subscript refers to ions; unsubscripted variables refer to electrons.) If energetic ion impact is the primary mechanism for release of electrons from the cathode, then

$$
-\gamma_{p} n_{+}(0) v_{+}(0)=n(0) u(0)
$$

Combining the two preceding equations to eliminate the ion flux, $n_{+} v_{+}$, the number density at the cathode is found to be

$$
n(0)=-(j / e) /\left[\left(1+1 / \gamma_{p}\right) u(0)\right] \text {. }
$$

A few comments are in order concerning the parametrization of secondary electrons liberated from the cathode by energetic ion impact. The coefficient $\gamma_{p}$ is only an estimate, and excludes other electron ejection mechanisms at the cathode such as metastable impact--see, for instance, Rundel et $a l .^{14}$ The coefficient $\gamma_{p}$ is not well known; the experiments of Doughty, and co-workers indicated a composite value of $\gamma_{p} \approx 0.3$ over the range of discharge current dersities studied here. ${ }^{2}$ This value was used in the single beam model.

Finally, Poisson's equation can be rewritten in terms of

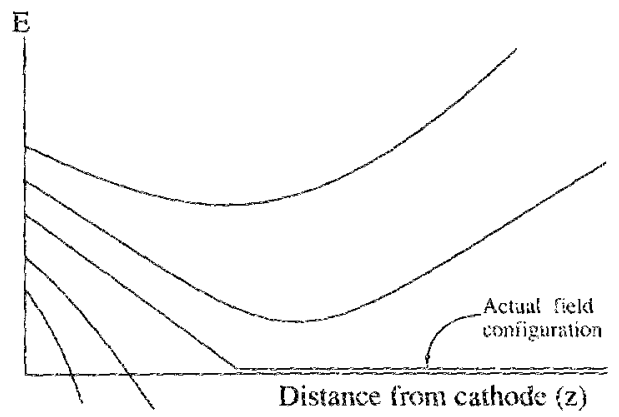

FIG. 1. A schematic representation of various electric field configurations, depending upon the choice for the electric field at the cathode $E_{\sigma}$.

known quantities using the expressions for current [Eq. (11)] and ion mobilicy [Eq. (10)]:

$$
\frac{d E}{d z}=\frac{e}{\varepsilon_{0}}\left(\frac{j / e+r u}{\mu_{+} E}-n\right)
$$

where $\mu_{+}$is defined in terms of the electric field in Eq. (10).

There are now three coupled equations $[(4),(6)$, and (14) $]$ in the three unknowns $n, u$, and $E$, that must be integrated to obtain the electron and electric field behavior in the cathode fall. Boundary conditions have been put on $n$ and $u$, and a family of solutions can be produced, depending upon the choice for the cathode electric field $E_{0}$. The family of electric configurations is shown schematically in Fig. 1. An additional condition must be placed on the electric field to single out the one choice for $E_{0}$ that is physically correct.

\section{E. Constraints on electric weld behavior}

In the most general sense, a discharge circuit can be viewed as a load line probiem. A schematic discharge circuit and its load line curve are presented in Fig. 2. The discharge will operate at a voitage and current corresponding to an intersection of its $V \cdot I$ characteristic with the load line. The hypothetical $V-I$ characteristic of Fig. 2 has multiple intersections with the load line. Intersection $A$ is metastable and will "decay" to C if disturbed; intersection B is dynamically unstable, and will also "decay" to C.

The physically correct electric field configuration is most conveniently chosen using an extreme load line. In the limit of an ideal voltage source $\left(Z_{B} \rightarrow 0\right)$, the load line be-

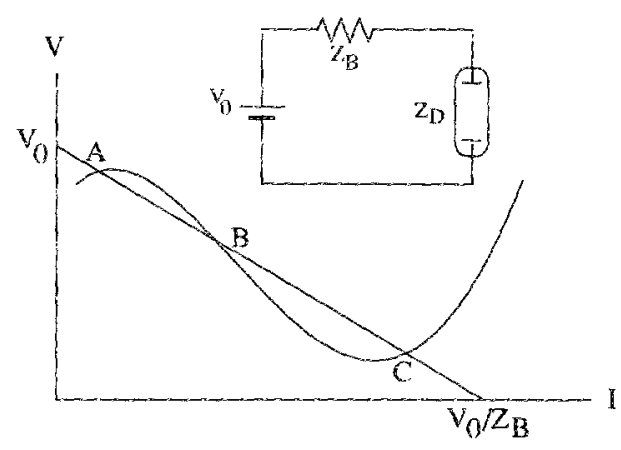

FIG. 2. Hypothetical discharge circuit and its associated load line diagram. The $D$ subscript refers to the dischesrge, and $D$ to the ballast impedence. 
comes horizontal and the discharge will maximize current in stable operation at fixed voltage. In the limit of an ideal current source $\left(V_{0} \rightarrow \infty, Z_{B} \rightarrow \infty\right.$, with $V_{0} / Z_{B}$ fixed), the discharge will minimize voltage in stable operation at fxed current.

In general, the most convenient of the above conditions may be used, as they are all equivalent, and all correspond to maximizing the entropy production within the discharge circuit. The assertion is supported by examining the rate of entropy production for the entire circuit of Fig. 2, which (in equilibrium systems for which we can define a temperature $T)$ is $d S / d t=I V_{0} / T{ }^{15}$ The entropy production is proportional to the power dissipated in the circuit, which is $P=I V_{0}=V_{0}^{2} /\left(Z_{B}+Z_{D}\right)$, where $Z_{D}$ is the discharge impedance. A condition of this kind was suggested by Compton and Morse ${ }^{16}$ in 1927 and reviewed by Druyvesteyn and Penning ${ }^{17}$ in 1940. Compton and Morse couched the principle of maximizing the entropy in the language of maximizing the ionization resulting from the launch of an electron from the cathode. It was subsequently used by others. ${ }^{18-20}$ Minimizing the voltage at fixed current is most convenient.

Referring to the family of possible electric field solutions (Fig. 1), the minimum possible potential drop would appear to be zero. In this case, however, the field curves that cross the $E=0$ axis are nonphysical solutions. The model is predicting an electron current at the zero-crossing point which does not correspond to the imposed current; an ionic current is implied. Since the parametrization of the ion behavior [Eq. (10)] implies $v_{+}=0$ in the absence of a field, an infnite ion density is predicted-a nonphysical solution. This is due to an inappropriate boundary condition, and is not attributable to the single beam model used here. Ensuring that all current is carried by the electrons at the cathode fall. negative glow boundary is necessary; this is one of the key results in Doughty and co-workers ${ }^{2}$ and Den Hartog and coworkers. ${ }^{4}$

The preceding paragraph does not imply that the electric field may never cross the $E=0$ axis, only that all current must be carried by the electrons whenever the field vanishes. Once the improper "zerc-crossing" solutions are eliminated, it is relatively easy to pick out the correct electric field behavior; it is the curve labeled as the "actual field configuration" in Fig. 1. Any of the curves that do not reach the axis will correspond to a higher discharge potential (immaterial of the integration limits) and can be eliminated. The solutions could also be eliminated because the single beam model implies an unphysical negative ion density at the boundary. However, this unphysical prediction is occurring exactly where the ion transport model is expected to fail--a lowfield region. The extremum condition constrains the behavior of the electric field without reference to a specific model.

\section{RESULTS}

The coupled equations were integrated using an explicit, fourth-order Runge-Kutta integration scheme. The collision cross sections were taken from LaBahn and Callaway ${ }^{21}$ (elastic) and Alkhazov ${ }^{22}$ (inelastic). Associative ionization was not included in the single beam model, and only excita-

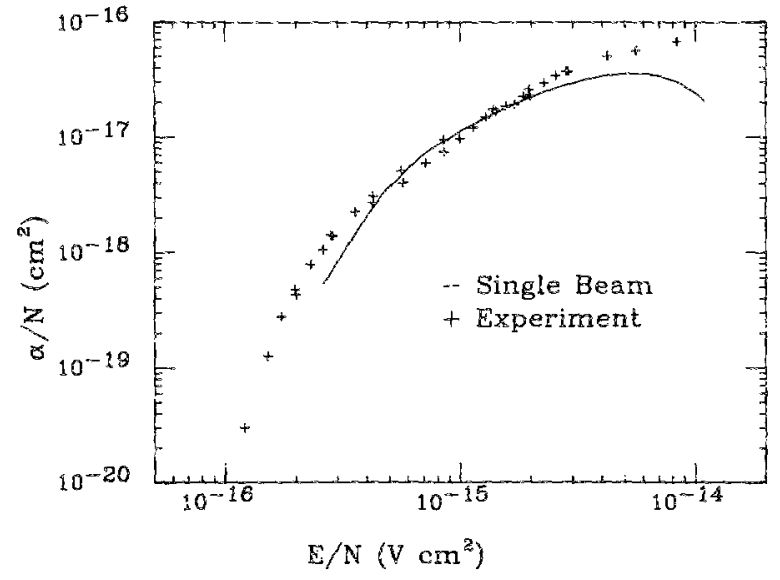

FIG. 3. Townsend's firse ionization coeficient for helium. The experimental points were compiled by Dutton (Ref. 23). The low $E / N$ start of the singie-beam prediction corresponds to the beam energy reaching ionization threshold.

tion to the six levels with the largest cross sections was considered.

To indicate the validity of the model used, it was first compared with known Townsend $\alpha$ values from various swarm experiments. ${ }^{23}$ The left-hand side of Eq. (5) was set to zero to obtain spatially invariant swarm results. The electric field was calculated for various drift velocities, and the Townsend $\alpha$ coefficient was then found. The results are shown in Fig. 3. The low-field limit of the single beam results is the field at which the beam energy equals the threshold ionization energy of helium; the model is obviously of marginal use at low (less than $3 \times 10^{-16} \mathrm{~V} \mathrm{~cm}^{2}$ in helium) electric fields. Above this threshold, however, the model's predictions are excellent for a decade of electric field values. The downturn of the predicted $\alpha$ at high fields shows the effect of the beam energy exceeding the peak of the ionization cross section. Further increases in the electric field actually reduce the collisional drag the beam feels. This contrasts with the real situation, where many electrons near the peak total collision cross section energy are scattered to lower energies, reaccelerated, and scatter again. The single-beam model would therefore seen to have a definite range of general application-from an electric field that produces a beam energy around the ionization threshold, to one where the beam energy is beyond the peak in the total inelastic cross section.

The model was then solved for some of the discharge parameters studied by Doughty and co-workers. Their experimental determination of the cathode fall electric field using optogalvanic techniques represents the state-of-the-art in noninvasive study of the cathode fall, ${ }^{24,25}$ and their simulations follow the null collision method of Boeuf and Marode. ${ }^{12}$ Considering the simplicity of the model, its predictions agree quite well with experimental resuits in the near-normal glow regime: however, it severely overestimates the cathode electric field and cathode fall length in the abnormal glow case. The predictions of the single-beam model rapidly deteriorate if the beam is accelerated greatly beyond 
TADLE I. Comparison of the single beam model with the experiments of Doughty and co-workers (Ref. 2 ). $V$ is the discharge potential, $d$ is the length of the cathode fall, and $E_{0}$ is the electric field at the cathode. "WM" refers to the direct volt-meter reading of the discharge potential, "OG" refers to the optogalvanic measurements, and " $\mathbf{B}$ " refers to the predictions of the single beam model. The number density is from Doughty. "Label" refers to the data presented in Figs. 47.

\begin{tabular}{|c|c|c|c|c|c|c|c|c|c|}
\hline \multirow[t]{2}{*}{ Label } & \multirow[t]{2}{*}{$\left(\mathrm{mAcm}^{-2}\right)$} & \multirow[t]{2}{*}{$\left(10^{36} \mathrm{~cm}^{-3}\right)$} & \multicolumn{3}{|c|}{$\stackrel{V}{(V)}$} & \multicolumn{2}{|c|}{$\begin{array}{c}d \\
(\mathrm{~cm})\end{array}$} & \multicolumn{2}{|c|}{$\begin{array}{c}E_{0} \\
\left(V \mathrm{~cm}^{-1}\right)\end{array}$} \\
\hline & & & VM & $0 G$ & 18 & OG & 13 & OG & $1 \mathbf{B}$ \\
\hline (a) & 0.190 & $\llbracket 1.2$ & 173 & 171 & 218 & 0.382 & 0.44 & 897 & 1010 \\
\hline (b) & 0.519 & 10.8 & 211 & 215 & 363 & 0.301 & 0.41 & 1426 & 1810 \\
\hline (c) & 0.846 & 10.3 & 261 & 264 & 651 & 0.282 & 0.50 & 1870 & 2700 \\
\hline
\end{tabular}

the energy where the various cross sections peak while the beam is still in a high-field region: in such a case, the collisional drag is almost zero and the electrons accelerate wildly. This behavior was hinted at in Fig. 3, where the Townsend $\alpha$ ionization coefficient was already underestimated by a factor of three at $E / N=10^{-14} \mathrm{~V} \mathrm{~cm}{ }^{2}$. An underestimation of the effective Townsend $\alpha$ corresponds to an overestimation of both the electric field and the thickness of the cathode fall region. Only representative results for discharges closest to the normal glow will be presented.

Experimental discharge parameters, along with some of the results of this model, are shown in Table 1. Figure 4 compares the electric field behavior as caiculated from the single-beam model and the optogalvanic results of Doughty and co-workers. ${ }^{2}$ The model faithfully predicts the dominantly linear spatial variation of the electric field observed in the case of a weakly ionized plasma, lending support to the use of an extremum condition to determine the appropriate electric field at the cathode. To facilitate comparisons in the low-field negative glow, a $10 \mathrm{~V} \mathrm{~cm}^{-1}$ electric field was imposed in the negative glow, and matched at the boundary to the calculated electric field in the cathode fall. As was noted with the predictions for the Townsend $\alpha$, the single beam model is of little use at the small fields existing in the negative

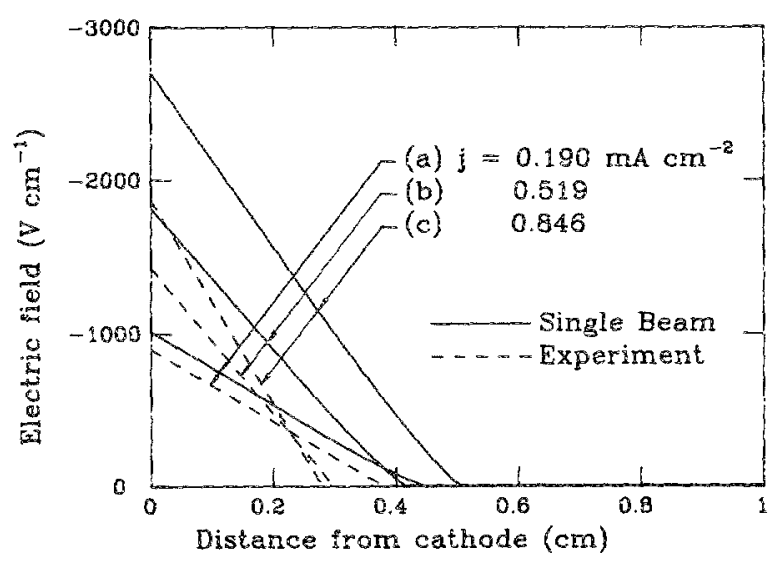

FIG. 4. Cathode fall electric field configuration predicted by the model, and the best least-squares fit to the optogalvanic data of Doughty and coworkers (Ref. 2). glow. In addition, there is the usual problem of numerically evaluating Poisson's equation [Eq. (8)], since $d E / d z$ (which is very small in the negative glow) is found by taking the difference between two very large numbers.

The spatial variation of the beam number density and energy are plotted in Figs. 5 and 6 , along with the number density and average energy results from Doughty's Monte Carlo calculations. ${ }^{3}$ These two parameters completely describe the distribution function, from which all other results may be calculated. Because Doughty used an imposed electric field for his Monte Carlo simulations (and ignored ion behavior), it is dificuit to make a direct numerical comparison of the calculated number densities. Here, the Monte Carlo number density at the cathode was simply scaled and set equal to the single beam number density at the cathode. Also, Doughty's simulations included the effect of a perfectly absorbing anode at a distance of $0.6 \mathrm{cr}$ from the cathode, while the single-beam results assumed a negative glow of infinite length. For this reason, Doughty's results reflect the absence of backscattered electrons in the neighborhood of the anode.

Figure 6 compares the model's beam exiergy and the Monte Carlo's average energy; only electron number and energy were balanced. Since results for, say, the effective Townsend $\alpha$ coefficient depend on absolute electron energy and not drift energy, it is perhaps not surprising that the

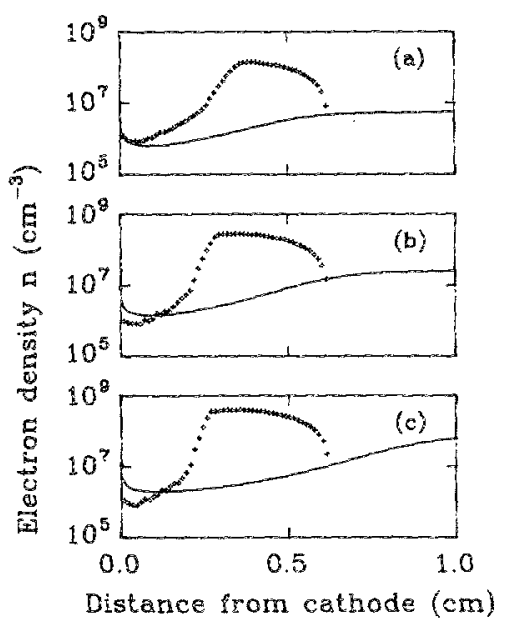

FIG. 5. Comparison of number density predictions from the Monte Carlo of Doughty (Ref. 3) and the single-bearn model here. The Monte Carlo results (crosses) were scaled to match the singie beam results (solid line) for cath. ode number density. 


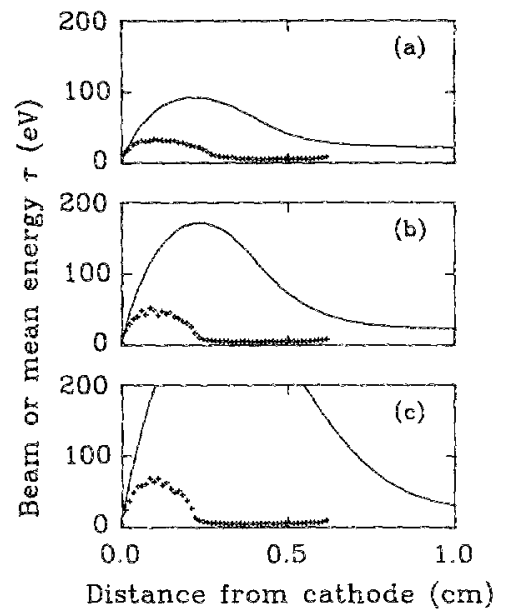

single beam would give reasonable results for $\alpha$ and electron energy, while seriously overestimating the average energy. Some work was done with the zeroth and first moments of the Boltzmann equation (electron number and momentum balance), which gave better velocity agreement on average, but underestimated the Townsend $\alpha$. Finally, the effective Townsend first ionization coeficient, $\alpha$, is plotted in Fig. 7 .

\section{SUMMARY}

A framework for modeling of the cathode fall region of a dc glow discharge with a self-consistent electric field has been developed. The electrons were treated by using a twoparameter beam model and the zerotk and second moments of the Boltzmann equation. Ion motion was accounted for using known mobilities for helium ions in neutral helium. The boundary conditions at the cathode were developed from experimentally known quantities. An additional condition was placed on the electric field to eliminate all solutions but the physically correct one. Some conditions were put on the range of $E / N$ where the model should be applied. The model was then solved for the discharge conditions of Doughty and co-workers. In spite of the simplicity of the model, the results were in good qualitative agreement with the experiments and simulations of Doughty and coworkers. A more sophisticated model distribution function solved within the framework presented here should produce better quantitative agreement with experiment.
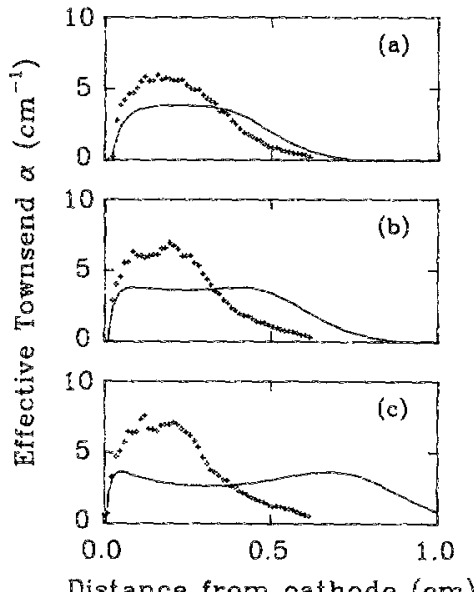

FIG. 7. Comparison of Townsend"s first ionization coefficient from the Monte Cario and the single-beam model. The motation is as in Fig. 5.

\section{ACKNOWLEDGMENTS}

This research is supported by the Air force Office of Scientific Research under Grant No. AFOSR 84-0328.

1P. Bayle, I. Vacquie, and M. Bayle, Phys. Rev. A 34, 360 (1986).

${ }^{2}$ D. A. Doughty, E. A. Den Hartog, and J. E. Lawler, Phys. Rev. Lett. 58, 2668 (1987)

${ }^{3}$ D. A. Doughty, Ph. D. thesis, University of Wisconsin (1987).

${ }^{4}$ E. A. Den Hartog, D. A. Doughty, and J. E. Lawler, Phys. Rev. A (in press).

5. H. Ingold, in Gaseous Electronics, edited by M. N. Hirsh and H. J. Oskam (Academic, New York, 1978), Vol. 1, Chap. 2, p. 37.

'A. V. Phelps, B. M. Jelenković, and L. C. Pitchford, Phys. Rev. A 36, 5327 (1987).

7J. E. Lawler, Phys. Rev. A 32, 2977 (1985).

'S. Sinha, S. L. Lin, and J. N. Bardsley, J. Phys. B 12, $16: 3$ (1979).

${ }^{3} \mathrm{H}$. Helm, J. Phys. B 10, 3683 (1977).

${ }^{10}$ L. S. Frost, Phys. Rey. 105, 354 (1957).

"G. H. Wannier, Statistical Physics (Wiley, New York, 1966), p. 462.

123. P. Boeuf and E. Marode, J. Phys. D 15, 2169 (1982).

13.T. N. An, E. Marode, and P. C. Johnson, J. Phys. D 10, 2317 (1977)

${ }^{14}$ R. D. Rundel, F. B. Dumning, J. S. Howard, J. P. Riola, and R. F. Stebbings, Rev. Sci. Instrum. 44,60 (1973).

${ }^{15}$ M. W. Zemansky and R. H. Dittman, Heat and Thermodynamics (McGraw-Hill, New York, [981), p. 205.

${ }^{16}$ K. T. Compton and P. M. Morse, Phys. Rev. 30, 305 (1927).

'M. J. Druyvesteyn and F. M. Penning, Rev. Mod. Phys. 12, 87 (1940),

${ }^{18 W}$. Rogowski, Z. Phys. 82, 473 (1933).

${ }^{19}$ A. von Hippel, $Z$. Phys. 76, $1(1932)$.

${ }^{26} \mathrm{~K}$. G. Emeleus, Int. J. Electron. 36, 1 (1974).

${ }^{2}$ R. W. LaBahn and J. Callaway, Phys. Rev. A 2, 366 (1970); Phys. Rev. 180, 91 (1969); Phys. Rev. 188, $\$ 20$ (1969).

${ }^{22}$ G. D. Alkhazov, Zh. Tekh, Fiz. 40, 97 (1970) [Soy. Phys. Tech. Phys. 15, $66(1970)]$.

${ }^{23}$ J. Dution, J. Phys. Chem. Ref. Data 4, 722 (1975).

${ }^{24}$ D. K. Doughty, S. Salih, and J. E. Lawler, Phys. Lett. A 103, 41 (1984).

${ }^{25}$ D. K. Doughty and J. E. Lawler, Appl. Phys. Lett. 45, 611 (1984). 